\title{
GERENCIAMENTO DE IMPRESSÃO NAS COMPANHIAS ABERTAS LISTADAS NA BRASIL, BOLSA, BALCÃO [B]: O USO DO TOM ANORMAL NOS RELATÓRIOS DE ADMINISTRAÇÃO
}

\author{
IMPRESSION MANAGEMENT IN PUBLIC COMPANIES LISTED ON THE NEW [B]³ \\ STOCK EXCHANGE: THE USE OF ABNORMAL TONE IN MANAGEMENT REPORTS
}

\section{Mariana de Oliveira Cavalheiro}

Mestra em Controladoria e Contabilidade pela Universidade Federal do Rio Grande do Sul (Porto Alegre/Brasil). Auditora de Controle Interno na Controladoria-Geral do Município (Porto Alegre/Brasil).

E-mail: mariana_ocavalheiro@hotmail.com

\section{Fernanda Gomes Victor}

Doutora em Administração pela Universidade Federal do Rio Grande do Sul (Porto Alegre/Brasil). Professora Permanente do Programa de Pós-Graduação em Contabilidade da Universidade Federal do Rio Grande (Rio Grande/Brasil) e do Programa de Pós-graduação em Controladoria e Contabilidade da Universidade Federal do Rio Grande do Sul (Porto Alegre/Brasil).

E-mail: fernanda.g.victor@gmail.com

\section{Arthur Frederico Lerner}

Doutorando em Contabilidade do Programa de Pós-Graduação em Contabilidade da Universidade Federal de Santa Catarina

(Florianópolis/Brasil).

E-mail: arthurlerner_@hotmail.com

\section{Rafaela Mâncio Grando}

Mestra em Controladoria e Contabilidade pela Universidade Federal do Rio Grande do Sul (Porto Alegre/Brasil). Assessora do departamento de Informações Contábeis da Companhia Riograndense de Saneamento - CORSAN (Porto Alegre/Brasil). E-mail: rafaela.grando@hotmail.com 


\section{RESUMO}

Cada vez mais há preocupações sobre se as informações retratadas nas narrativas contábeis descrevem fatos verdadeiros, ou se foram manipuladas de tal forma a influenciar as percepções dos leitores. Nesse sentido, o objetivo da pesquisa é analisar as características do gerenciamento de impressão por meio do uso de tom anormal nos relatórios de administração das companhias abertas listadas na Brasil, Bolsa, Balcão [B] ${ }^{3}$, durante os anos de 2010 a 2017. Para tanto, foi realizado um estudo com abordagem do problema quantitativa, por meio documental com análise descritiva dos dados. Ainda, 1.576 relatórios de administração foram coletados manualmente no site da CVM. O tom anormal, proxy para o gerenciamento de impressão, foi mensurado segundo o estudo de Carlsson e Lamti (2015) e, após, foi analisada a sua relação com a performance financeira por meio de regressão com dados em painel com efeitos aleatórios. Os principais resultados evidenciaram que a probabilidade de se observar o uso de um tom anormal positivo (gerenciamento de impressão positivo) nos relatórios de administração diminui conforme o desempenho financeiro das empresas diminui. Espera-se que o presente trabalho permita um maior conhecimento sobre essa prática de gerenciamento e, dessa forma, os usuários consigam detectá-la mais facilmente.

Palavras-chave: Gerenciamento de impressão. Gerenciamento da informação. Tom anormal. Relatório de administração.

\section{ABSTRACT}

Increasingly, there are concerns about whether information portrayed in accounting narratives describes true facts, or whether they have been manipulated in a way that influences readers' perceptions. In this sense, this research aims to analyze the characteristics of impression management through the use of abnormal tone in management reports of public companies listed in Brasil, Bolsa, Balcão [B] ${ }^{3}$, during the years of 2010 to 2017. In order to do so, a study with quantitative problem approach was carried out through documentary analysis with descriptive data analysis. Still, 1,576 management reports were manually collected on the CVM portal. The abnormal tone, proxy for impression management, was measured according to the study of Carlsson and Lamti (2015) and, afterwards, its relation with the financial performance was analyzed through regression with panel data with random effects. The main results showed that the probability of observing the use of an abnormal positive tone (positive impression management) in management reports diminishes as the financial performance of companies decreases. It is expected that the present work will allow a greater knowledge about this management practice and, in this way, the users will be able to detect it more easily.

Keywords: Impression management. Information management. Abnormal tone. Management report. 


\section{INTRODUÇÃO}

No contexto da contabilidade, os usuários geralmente associam divulgações financeiras somente a números (CARLSSON; LAMTI, 2015). Contudo, as narrativas contábeis, que são aquelas que não apresentam informações numéricas ou tentam explicá-las, representam um meio de comunicação financeira cada vez mais importante (CLATWORTHY; JONES, 2003). Cunha e Silva (2009) afirmam que elas podem ser encontradas em pronunciamentos de entidades reguladoras, processos legais e em relatórios corporativos, como, por exemplo, nos relatórios de administração. Todavia, cada vez mais há preocupações sobre se as informações retratadas nessas narrativas descrevem fatos verdadeiros, ou se foram manipuladas de tal forma a influenciar as percepções dos leitores e, eventualmente, suas decisões de investimento (CEN; CAl, 2013).

Neste contexto, Cunha e Silva (2009) ressaltam que na maioria dos textos divulgados ao mercado não passam por revisões da auditoria, criando oportunidade aos administradores, de manipular tais informações. Assim sendo, esse comportamento dos gestores que buscam explorar a assimetria de informação entre eles e os usuários externos através da manipulação de relatórios é chamado de gerenciamento de impressões (MERKL-DAVIES; BRENNAN, 2007).Na maioria das vezes, esse tipo de prática está associada à evidenciação voluntária da empresa, quando a entidade divulga as informações com o objetivo principal de construir a sua imagem corporativa de forma positiva e de tentar influenciar a opinião dos stakeholders (BONFIM et al., 2018).

Estudos sobre gerenciamento de impressão na área da contabilidade geralmente investigam manipulações dos administradores em gráficos (BEATTIE; JONES, 1992; MATHER et al., 2005; JONES, 2011; CHO et al., 2012; FALSCHLUNGER et al., 2015) e em narrativas contábeis (HENRY, 2008; HUANG et al., 2014; PATELLI; PEDRINI, 2014; BOZZOLAN et al., 2015; SAORIN et al., 2017). Dentre essas duas maneiras, o foco da presente pesquisa é analisar o tom utilizado nas narrativas contábeis dos relatórios de administração da amostra.

Esse tipo de relatório contábil tem forte poder de comunicação, segundo Silva et al. (2007), pois ele é menos técnico e mais descritivo do que outros documentos. Também, ele é utilizado pelas entidades para prestar contas dos atos praticados pela companhia e para apresentar expectativas sobre desempenhos futuros. Dessa forma, é importante analisar a forma com que os discursos dos administradores são divulgados nesse relatório, tendo em vista que eles são considerados como enviesados por boa parte da literatura (SILVA et al., 2007).

Isso posto, o presente estudo dedica-se a responder à seguinte questão: Quais as características do gerenciamento de impressão por meio do uso de tom anormal nos relatórios de administração das 
companhias abertas listadas na Brasil, Bolsa, Balcão $[B]^{3}$ ? Nesse sentido, a pesquisa tem como objetivo analisar as características do gerenciamento de impressão por meio do uso de tom anormal nos relatórios de administração das companhias abertas listadas na [B] $]^{3}$ durante os anos de 2010 a 2017.

No âmbito nacional, Miranda et al. (2008), Cunha e Silva (2009), Maior et al. (2012), Penteado (2013), Souza (2017) e Bonfim et al. (2018) realizaram estudos sobre o tema. Miranda et al. (2008), Maior et al. (2012), Penteado (2013) e Bonfim et al. (2018) avaliaram a prática de gerenciamento de impressão por meio de distorções em gráficos. Por outro lado, Cunha e Silva (2009) e Souza (2017) tiveram sua análise nas narrativas contábeis, sendo que este avaliou o tom linguístico utilizado pelas companhias de sua amostra e aquele mediu o grau de facilidade de leitura nos relatórios de administração e nas notas explicativas.

É válido salientar que, apesar de ambas as pesquisas investigarem o tom utilizado nas narrativas contábeis, o presente artigo se diferencia daquele proposto por Souza (2017) em importantes aspectos. Souza (2017), diferentemente deste artigo, não se utilizou de dicionário de palavras, realizando manualmente a codificação e análise das mesmas. Isso se deu pois sua amostra contou com apenas 47 empresas, enquanto que a do presente estudo é composta por 167 companhias, inviabilizando a análise manual das mesmas. Ainda, a diferença principal entre esta pesquisa e a de Souza (2017) é que o autor não dividiu a análise de tom em normal e anormal, enquanto que, neste estudo, a proxy do gerenciamento de impressão é apenas o tom anormal apresentado.

A pesquisa busca contribuir para a literatura a partir do fomento a discussão sobre o gerenciamento de impressão, demonstrando como este pode ocorrer no desenvolvimento das informações divulgadas ao mercado. De forma prática, o estudo almeja demonstrar ao mercado a importância da análise dos discursos empregados nos relatórios de administração, evidenciando que a real situação da empresa pode ser melhor diagnosticada a partir da análise do seu desempenho divulgado em demonstrações financeiras e notas explicativas e em conjunto com a análise da narrativa dos relatórios descritos.

\section{REFERENCIAL TEÓRICO}

\subsection{GERENCIAMENTO DE IMPRESSÃO NA CONTABILIDADE}

Segundo Schlenker (1980), o gerenciamento de impressão é uma atividade que tem como objetivo controlar ou regular informação para influenciar as impressões formadas pelos leitores. Seu conceito teve origem na psicologia social, mas, na Contabilidade, está relacionado à manipulação do conteúdo nas narrativas contábeis ou à distorção na apresentação de ilustrações nos relatórios corporativos, como 
gráficos ou imagens (BEATTIE; JONES, 2000). Também, ele é explicado pela Teoria da Agência como sendo o viés utilizado nas divulgações da empresa e como sendo o comportamento oportunista dos gerentes, que selecionam um estilo de apresentação e de conteúdo que seja benéfico para eles (BEATTIE; JONES, 2000).

De acordo com Michelon (2012), a construção da imagem corporativa, e consequentemente da legitimação da organização, é a maior motivação para os administradores gerenciarem a impressão das companhias. A organização está inserida em um espaço social que é composto por diversas exigências e padrões socioculturais esperados pela sociedade. Mendonça e Amantino-de-Andrade (2003) defendem que, nesse contexto, o gerenciamento de impressão ocorre por meio de uma comunicação, na qual são enviadas mensagens sobre a entidade, sejam reais ou fictícias, para atender à demanda da sociedade. Nesse sentido, Hines (1988) explica como os administradores acabam por criar uma realidade na contabilidade, ao invés de simplesmente representá-la. Segundo o autor, é esperado desses administradores que eles comuniquem a realidade. Contudo, as pessoas já têm uma certa concepção da realidade. Por isso, os comunicadores acabam por refletir essa realidade criada pela sociedade para atender às suas expectativas. Pois, caso contrário, a credibilidade da empresa seria prejudicada (HINES, 1988). Esse processo está relacionado à formação e à manutenção da imagem corporativa, que, por consequência, impacta a legitimação dessas organizações.

Caso a entidade tenha êxito em atentar às demandas da sociedade, conseguindo conquistara legitimação em seu ambiente, ela passa a contar com os recursos econômicos e simbólicos que são necessários à sua sobrevivência (MENDONÇA; AMANTINO-DE-ANDRADE, 2003). Em alguns casos, após esse processo, a organização pode até influenciar os padrões socioculturais do ambiente no qual está inserida. Por isso, o processo de gerenciamento de impressão apresentado pelos autores é contínuo, visto que as empresas estão constantemente buscando legitimidade e sendo afetadas pelos seus stakeholders.

De acordo com Saorin e Osma (2010), existem diferentes técnicas empregadas nas narrativas dos gestores da organização que poderiam levar a percepções equivocadas sobre elas. Os autores afirmam que essa prática pode ocorrer nos relatórios anuais ou ainda em comunicados informais à imprensa. Isso se dá, pois essas informações que são divulgadas à imprensa permitem um alto nivel de discricionariedade. Elas incluem informações que não são reportadas nas demonstrações financeiras (tanto qualitativas quanto quantitativas), o que permite práticas estratégicas de divulgação (SAORIN; OSMA, 2010).

Para Merkl-Davies e Brennan (2007), existem dois comportamentos que podem ocorrer no gerenciamento de impressão: o de ocultação ou o de atribuição. Os autores explicam que a ocultação pode acontecer de duas maneiras: ofuscando, omitindo ou disfarçando resultados e notícias negativas ou ainda 
ressaltando ou enfatizando resultados ou notícias positivas. Por outro lado, defendem que a atribuição é uma tática defensiva, na qual os administradores tentam afastar a culpa de resultados negativos de si.

Em se tratando da ocultação, Merkl-Davies e Brennan (2007) apresentaramalgumas estratégias para gerenciar a impressão da empresa. Para esconder resultados negativos, é comum que os administradores tornem a leitura do relatório mais difícil (menos compreensivel) ou utilizem uma linguagem persuasiva. Para ressaltar os resultados positivos, eles podem: i) utilizar um viés otimista, enfatizando palavras otimistas, ii) ressaltar a apresentação visual ou mudar a ordem das informações, iii) comparar seus resultados com outras empresas que estejam em situação pior, para que a imagem da empresa seja a melhor possivel; ou iv) selecionar números positivos do resultado financeiro para divulgação no relatório (MERKL-DAVIES; BRENNAN, 2007). Para observar o uso desse tipo de gerenciamento, é possível fazer uma análise do uso de uma linguagem positiva ou negativa na narrativa dos relatórios das organizações. Essa análise consiste em classificar as palavras em positivas ou negativas para inferir a possibilidade de existência de manipulação (SAORIN; OSMA, 2010). Outra prática observável do gerenciamento é quando há uma repetição de informações ou quando partes específicas da informação são reforçadas para que elas se destaquem mais aos leitores (BRENNAN et al., 2009).

\subsection{ESTUDOS RELACIONADOS}

No âmbito internacional, mais de 40 estudos sobre gerenciamento de impressão foram encontrados. Contudo, como a presente pesquisa tem como amostra as companhias listadas na [ $\mathrm{B}^{3}$ ], o foco da revisão de literatura nesta seção paira sobre os estudos nacionais. Portanto, no contexto do Brasil, foram localizadas seis pesquisas sobre o tema.

A pesquisa nacional pioneira na área foi a de Miranda et al. (2008), que replicaram o estudo internacional de Beattie e Jones (1992). Os autores brasileiros investigaram se os gráficos ilustrados nos relatórios que apresentam bom desempenho são apresentados de maneira diferente quando comparados aos gráficos dos relatórios que apresentam prejuízo. Analisaram 37 relatórios de 17 companhias abertas listadas na bolsa de valores de São Paulo, na época denominada Bovespa. Para verificar as características dos gráficos, Miranda et al. (2008) analisaram a quantidade de gráficos apresentados no relatório, a variável apresentada, o formato do gráfico e a área de cada gráfico apresentado. Após, os dados das empresas que apresentaram prejuízo foram comparados com os das empresas que obtiveram lucro. Seus achados indicam que as companhias com situação lucrativa apresentam gráficos em quantidade maior, com variáveis diferentes e dedicam maior área a representações gráficas do que as que apresentam prejuízo. 
Cunha e Silva (2009), por outro lado, investigaram o gerenciamento de impressão nas notas explicativas e relatórios da administração das empresas listadas na Bovespa por meio das narrativas contábeis. A partir da fórmula de Flehs, os autores mediram o tamanho das palavras e a quantidade delas por sentença e a partir disto, realizaram análises sobre o grau de facilidade de leitura, isto é, se os textos contidos nesses documentos são mais ou menos complexos para gerenciarem as informações sobre o seu desempenho. A partir das variáveis de desempenho (variação no preço das ações, lucro, ativo e receita), os achados de Cunha e Silva (2009) indicaram que o grau de facilidade dos textos analisados pode ser classificado como difícil, sendo que a leitura das notas explicativas foi considerada como mais complexa (e técnica) do que a dos relatórios de administração (CUNHA; SILVA, 2009). Por fim, foram encontradas fracas evidências de gerenciamentos de impressões nas narrativas estudadas, contudo, evidenciaram uma relação entre o porte da empresa, o crescimento contínuo e a melhora na facilidade de leitura de seus textos.

Maior et al. (2012) realizaram uma pesquisa experimental, buscando compreender o que é o gerenciamento de impressão e qual a sua influência no processo decisório, tendo como base distorções nos gráficos. 0 experimento foi realizado com 267 estudantes de graduação dos cursos de contabilidade e áreas correlatas com a apresentação de duas empresas fictícias. Para tanto, foram aplicados três modelos de questionários, em que cada estudante deveria escolher quanto investir em cada uma das alternativas de investimento (empresa A e empresa B) (MAIOR et al., 2012). Os achados da pesquisa de Maior et al. (2012) mostram que mesmo quando os gráficos da empresa B eram distorcidos para apresentarem uma tendência mais positiva, os estudantes optaram por investir mais na empresa A. Dessa forma, os autores demonstram que essa escolha (de investir mais na primeira opção) pode indicar um efeito associado à ordem em que as opções de investimento foram apresentadas.

Penteado (2013), com o objetivo de observar a utilização dos gráficos apresentados anos relatórios de sustentabilidade como ferramenta para o gerenciamento de impressão, a partir de cinquenta companhias brasileiras de capital aberto, analisou 3.422 gráficos. No estudo, os indícios de gerenciamento foram observados por meio de duas técnicas apontadas: a seletividade e a distorção de medidas nos gráficos. Em relação à seletividade, foi identificado que a quantidade de gráficos para reportar notícias boas foi significativamente superior à quantidade de gráficos para reportar notícias ruins, não sendo possível inferir que houve uma preferência deliberada por parte das organizações para dar destaque às notícias positivas. Em se tratando da distorção das medidas nos gráficos, Penteado (2013) demonstrou que as quantidades de distorção dos gráficos para melhor e para pior foram muito próximas, por isso, não pode inferir que houve gerenciamento de impressão por meio dessas distorções. 
Bonfim et al. (2018) realizaram estudo muito similar ao de Maior et al. (2012), buscando também verificar se a forma como os gráficos com informações contábeis é apresentada pode influenciar o processo decisório dos estudantes de contabilidade. Bonfim et al. (2018) também aplicaram questionário com o objetivo de averiguar em qual empresa os estudantes iriam preferir investir, considerando somente os gráficos demonstrados. Contrariando os resultados de Maior et al. (2012), Bonfim et al. (2018) inferiram que o gerenciamento de impressão influenciou na tomada de decisão dos respondentes da sua amostra.

A pesquisa de Souza (2017) analisou o uso do tom nas narrativas contábeis. O autor buscou responder de que maneira o tom linguístico empregado nos discursos durante as conferências de apresentação de resultados está relacionado ao desempenho das empresas. Sua base de dados foi composta por 837 conferências de resultados em que 47 empresas participaram. Para avaliar o tom linguístico, Souza (2017) contou a frequência de vezes em que cada palavra (positiva ou negativa) apareceu e usou a fórmula de Henry (2008) para encontrar a pontuação do tom nos discursos. A principal diferença da presente pesquisa é que Souza (2017) não separou o tom em normal e anormal, e ainda, diferentemente desta pesquisa, Souza (2017) optou por não utilizar bancos de palavras com classificações prévias de otimismo ou pessimismo. Ele escolheu, portanto, codificar as palavras manualmente para realizar a contagem das mesmas. Seus resultados indicaram que o tom aparece relacionado com o desempenho presente e algum desempenho futuro, porém, que ele não tem o poder de predizer resultados.

A principal crítica em relação ao uso de banco de palavras realizada por Souza (2017) é de que não há bancos de palavras de estudos anteriores em português, somente em inglês. Na presente pesquisa, para suprir essa possivel inconsistência, o dicionário de palavras traduzido dos estudos de Henry (2008) e Carlsson e Lamti (2015) foi enviado para 3 profissionais brasileiros envolvidos com a elaboração e/ou leitura dos relatórios de administração, solicitando a validação desse dicionário.

Henry (2008) e Merkl-Davies e Brennan (2007) concluíram em seus estudos que o tom nos relatórios é gerenciado dependendo se as empresas estão tendo pouco ou muito lucro, indicando, ainda, que quando o lucro está alto, os gerentes tendem a usar um tom positivo nas narrativas contábeis como resultado de uma boa gerência (CARLSSOM; LAMTI, 2015). Ainda, Huang et al. (2014) defendem que quando a divulgação da informação é neutra, o otimismo no tom é positivamente associado à performance da companhia. Assim, formulou-se a Hipótese $1\left(\mathrm{H}_{1}\right)$ :

$\mathrm{H}_{1}$ : O tom total nos relatórios de administração é positivamente associado à performance financeira da empresa. 
Por outro lado, Carlsson e Lamti (2015) apresentam a pesquisa de Rutherford (2005) para dissertar sobre o uso do tom anormal positivo (gerenciamento de impressão positivo) em relação à performance financeira. Nela, o autor evidencia que empresas que apresentam prejuízo usam as palavras "lucro" e "lucrar" mais do que empresas que apresentam lucro. Com isso, o uso do tom anormal positivo nos relatórios estaria positivamente associado a um resultado negativo.

Ainda, Huang et al. (2014) defendem que empresas com prejuízo têm incentivos para mudar as percepções dos usuários, de forma que eles não consigam identificar o momento ruim da companhia. Com isso, essas empresas estariam mais propensas a empregar o gerenciamento de impressão positivo (tom anormal positivo) em suas narrativas. Isso posto, a Hipótese $2\left(\mathrm{H}_{2}\right)$ é, então, apresentada.

$\mathrm{H}_{2}$ : A probabilidade de se observar o uso de um tom anormal positivo nos relatórios de administração aumenta conforme o desempenho financeiro das empresas diminui.

\section{PROCEDIMENTOS METOdOLÓGICOS}

Com a finalidade de analisar as características do gerenciamento de impressão por meio do uso de tom anormal nos relatórios de administração das companhias abertas listadas na $\left[\mathrm{B}^{3}\right]$, foi realizado um estudo qualitativo-quantitativo (RAUPP; BEUREN, 2014) considerando-se que apesar da análise minuciosa dos relatórios de administração ser necessária, cada palavra analisada recebeu uma pontuação para, posteriormente, serem utilizados instrumentos matemáticos para o cálculo do gerenciamento de impressão. Quanto aos objetivos, a pesquisa é classificada como descritiva, pois busca descrever as características das empresas analisadas e no que se refere ao procedimento utilizado para a coleta dos dados, a pesquisa classifica-se como documental, partindo-se de documentos primários, ainda não analisados pela academia (GIL, 2008; MARTINS, 2013).

A população da pesquisa compreende as companhias abertas listadas na [B³]. Para compor a amostra, foram excluídas as empresas que não divulgaram as informações necessárias à realização do artigo. Portanto, o processo de amostragem foi do tipo não probabilístico intencional (COLAUTO, BEUREN, 2014). 0 procedimento de coleta para a realização desta pesquisa se deu de forma manual por meio do portal eletrônico da CVM. Como 167 empresas foram estudadas ao longo de 8 anos, 1.336 relatórios de administração foram baixados, manualmente, diretamente do portal da CVM. A Tabela 1 ilustra a formação da amostra. 


\begin{tabular}{lc}
\multicolumn{2}{c}{ Tabela 1 - Amostra } \\
\hline População total & $\mathbf{3 6 8}$ \\
( - ) Empresas com dados financeiros faltantes & 122 \\
( - ) Empresas com problemas nos R.A & 2 \\
( - ) Empresas do setor financeiro & 47 \\
( - ) Outliers & 30 \\
\hline Amostra do estudo & 167 \\
\hline \multicolumn{2}{c}{ Fonte: Elaborada a partir dos dados da pesquisa (2018). } \\
R.A = Relatórios de Administração
\end{tabular}

Dessa maneira, a população era composta de 368 empresas. Em seguida, 122 empresas foram excluídas por não apresentarem os dados financeiros necessários ao estudo. Adicionalmente, duas companhias foram excluídas por problemas nos relatórios de administração. Finalmente, 47 empresas do setor financeiro foram descartadas da amostra para não enviesar a pesquisa, tendo em vista que são regidas por regras contábeis específicas (DOMINGOS et al., 2017). Por fim, 30 empresas outliers foram excluídas. Sendo assim, a amostra final da pesquisa foi composta por 167 entidades.

Para identificar o gerenciamento de impressão, as informações contidas nos relatórios de administração foram analisadas por meio da análise de conteúdo. Segundo Severino (2007), na análise de conteúdo procura-se compreender, de forma crítica, o sentido manifesto ou oculto das comunicações. A proxy para o gerenciamento de impressão utilizada nessa pesquisa foi o tom anormal das palavras nos relatórios de administração. Segundo Carlsson e Lamti (2015), o tom nas narrativas contábeis é constituído de componentes "normais" e "anormais". Os componentes anormais, no presente estudo, foram calculados a partir dos resíduos (e) da Equação (2). Dito isso, o tom anormal funciona nesse estudo como proxy do gerenciamento de impressão (CARLSSON; LAMTI, 2015).

Para o cálculo do tom nos relatórios fez-se necessária a contagem das palavras classificadas como negativas e positivas, tendo as mesmas sido definidas por meio de um dicionário de palavras. A versão inicial do dicionário utilizado foi aquela proposta por Henry (2008) e, posteriormente complementada por Carlsson e Lamti (2015). O dicionário foi composto por uma lista de palavras classificadas em positivas e em negativas em inglês. Posteriormente, as palavras do dicionário de Henry (2008) e Carlsson e Lamti (2015) foram traduzidas para o português, representando uma versão base que foi posteriormente submetida à validação.

Para manter o rigor científico da pesquisa e tornar o dicionário o mais completo possível, o processo de validação do questionário deu-se por meio do envio de e-mail para três profissionais envolvidos com a 
elaboração e/ou leitura dos relatórios de administração solicitando a sua análise. O e-mail foi enviado para um professor/pesquisador de Contabilidade, para um analista de investimentos e para um gerente de relações com investidores de uma multinacional. O objetivo do processo de validação foi excluir palavras fora do contexto brasileiro, e, ainda, inserir possiveis palavras faltantes.

Com o dicionário em mãos, a contagem das palavras classificadas em positivas e em negativas nos relatórios de administração foi realizada com o auxílio do software de análise de conteúdo MAXQDA 12, que permite que todos os relatórios sejam inseridos no programa e analisados individualmente. Finalmente, para o cálculo do tom nos relatórios de administração, foi utilizada a Equação (1), elaborada por Henry (2008).

\section{TOM $=\frac{\text { No PalavrasPositivas }-N \text { o PalavrasNegativas }}{N \text { o PalavrasPositivas }+N \text { o PalavrasNegativas }}(1)$}

A Equação (1) possui uma escala com intervalos entre -1 e +1 . Se o resultado da equação for igual a 0 , significa que há indícios de que o relatório de administração foi construído de forma neutra. Por outro lado, um resultado entre -1 e 0 sugere que foi utilizado um maior número de palavras negativas. Já, um resultado entre 0 e +1 sugere que foram usadas mais palavras positivas (CARLSSON; LAMTI, 2015). Após encontrado o índice, ele foi analisado descritivamente e avaliado por setor, bem como foi realizada uma análise das palavras (positivas e negativas) mais utilizadas.

Huang et al. (2014) afirmam que o tom total (TOM) de uma narrativa é interpretado como a soma do tom normal com o tom anormal (TA). Um tom normal simboliza uma descrição neutra dos fundamentos da empresa e é expresso por meio das variáveis de controle da Equação (2). Dessa forma, a variável TOM (tom total) expressa o nivel de tom nos relatórios incluindo tanto o tom normal quanto o tom anormal (TA). Assim sendo, com a finalidade de avaliar se o tom total nos relatórios de administração é positivamente associado à performance financeira da empresa, conforme a $\mathrm{H}_{1}$ proposta, o tom anormal, proxy do gerenciamento de impressão neste estudo, é encontrado a partir dos resíduos da Equação (2), com base em Carlsson e Lamti (2015).

$$
T^{T O M} M_{i, t}=\beta_{0}+\beta_{1} R E N T_{i, t}+\beta_{2} T_{A M} M_{i, t}+\beta_{3} P_{-} V P A_{i, t}+\beta_{4} R E T_{i, t}+\beta_{5} S E T O R_{i, t}+\varepsilon_{i, t}(2)
$$

Em que: TOM $_{i, t}=$ tom total (mensurado a partir da Equação 3) da empresa $i$ no ano $t ;$ RENT $_{i, t}=$ rentabilidade (divisão do lucro líquido pelo ativo total) da empresa $i$ no ano $t_{i} \operatorname{TAM}_{i, t}=$ tamanho da empresa $i$, representado pelo logaritmo natural do ativo total $i$ no ano $t_{i} P_{-} V P A_{i, t}=$ preço da ação/ valor patrimonial da ação da empresa $i$ no ano $t_{i} R E T_{i, t}=$ retornos anuais das ações (Preço da ação $t_{1}$-Preço da ação $t_{0} /$ Preço 
da ação $t_{0}$ ) da empresa $i$ no ano $t_{i}$ SETOR ${ }_{i, t}=$ setor econômico da empresa $i$ no ano $t$, representado por variáveis dummies; $\varepsilon_{i, t}=$ resíduos da regressão - tom anormal $-\mathrm{TA}$.

Neste contexto, ressalta-se os achados de Huang et al. (2014), que indicam que empresas com prejuízo têm incentivos para mudar as percepções dos usuários, sendo propensas a empregar o gerenciamento de impressão positivo (tom anormal positivo) em suas narrativas. Assim sendo, para testar a $\mathrm{H}_{2}$ proposta na presente pesquisa, o modelo de regressão logística, representado pela Equação (3), também baseado no estudo de Carlsson e Lamti (2015) é realizado:

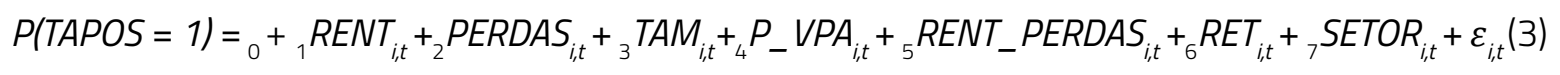

Em que, TAPOS $_{i, t}=$ dummy (em que para tom anormal (TA) positivo, igual a 1 e zero caso contrário) da empresa $i$ no ano $t_{i} R E N T_{i, t}=$ rentabilidade (divisão do lucro líquido pelo ativo) da empresa $i$ no ano $t_{i}$ PERDAS $_{i, t}=$ dummy (em que se RENT<0 igual a 1 e zero caso contrário) da empresa $i$ no ano $t_{i}$ TAM $_{i, t}$ $=$ tamanho da empresa $i$ representado pelo logaritmo natural do ativo total $i$ no ano $t_{i} P_{-} V P A_{i, t}=$ preço da ação/valor patrimonial da ação da empresa $i$ no ano $t_{i} R E N T_{-} P E R D A S_{i, t}=$ variável de interação entre rentabilidade e perdas da empresa $i$ no ano $t_{i} \mathrm{RET}_{i, t}=$ retornos anuais das ações da empresa $i$ no ano $t_{\text {; }}$ $\mathrm{SETOR}_{i, t}=$ setor econômico da empresa $i$ no ano $t$, representado por variáveis dummies; $\varepsilon_{i, t}=$ resíduos da regressão.O modelo de regressão logística representado pela Equação (3) possibilita a predição de valores tomados por uma variável categórica, dummy, a partir de uma série outras variáveis explicativas, que podem ser binárias ou contínuas (WOOLDRIDGE, 2002).

Após a revisão de literatura, é possivel explicar as razões da utilização de cada variável de controle do modelo. As variáveis rentabilidade (RENT) e perdas (PERDAS) captam o desempenho financeiro atual da empresa (CARLSSON; LAMTI, 2015) e, estudos como os de Huang, Teoh e Zhang (2014) e Davis e Tama-Sweet (2012) confirmam que a performance financeira da empresa afeta o nível do tom usado nos relatórios de administração.

A variável representativa do tamanho das companhias se justifica, pois Li (2010) afirma que empresas maiores tendem a ser mais cuidadosas ao se expressarem para evitar custos legais e políticos. As variáveis representativas do potencial de crescimento das empresas, preço da ação sobre valor patrimonial da ação (P_VPA) e Retornos anuais das ações (RET) também são coerentes com o modelo, tendo em vista que gerentes de empresas em alto crescimento têm mais incentivos para reportar as informações de forma estratégica, influenciando o tom usado (DAVIS; TAMA-SWEET, 2012). 
Por fim, a variável de interação da rentabilidade com as perdas (RENT_PERDAS) foi utilizada, pois Carlsson e Lamti (2015) defendem que como a variável PERDAS é dependente da variável RENT, surge um efeito de interação. Por isso, essa variável foi utilizada para controlar esse efeito.

\section{APRESENTAÇÃO E ANÁLISE dOS RESULTADOS}

\subsection{ANÁLISE POR SETOR}

A amostra é composta por 167 companhias abertas, observadas ao longo de oito anos (20102017). Na Tabela 2, é evidenciada a distribuição dessas 167 empresas estudadas entre setores, de acordo com a classificação disponível da [B³].

\begin{tabular}{lcc}
\multicolumn{3}{c}{ Tabela 2 - Distribuição de amostra entre setores } \\
\hline Setor & $\mathbf{N}^{\mathbf{0}}$ & $\%$ \\
Bens Industriais & 31 & $18,56 \%$ \\
Consumo Cíclico & 46 & $27,54 \%$ \\
Consumo Não Cíclico & 14 & $8,38 \%$ \\
Materiais Básicos & 24 & $14,37 \%$ \\
Petróleo, Gás e Biocombustíveis & 3 & $1,80 \%$ \\
Saúde & 9 & $5,39 \%$ \\
Tecnologia da Informação & 3 & $1,80 \%$ \\
Telecomunicações & 3 & $1,80 \%$ \\
Utilidade Pública & 34 & $\mathbf{2 0 , 3 6 \%}$ \\
\hline Total & $\mathbf{1 6 7}$ & $\mathbf{1 0 0 , 0 0 \%}$ \\
\hline \multicolumn{1}{c}{ Fonte: Elaborada a partir dos dados da pesquisa (2018). }
\end{tabular}

De acordo com a Tabela 2, o setor mais representativo da amostra, de acordo com a classificação da $\left[\mathrm{B}^{3}\right]$, foi o de Consumo Cíclico, correspondendo a 27,54\% da amostra. Em segundo lugar, o setor denominado Utilidade Pública representou cerca de $20 \%$ do conjunto estudado. Com aproximadamente $18 \%$ de representatividade, o setor de Bens Industriais foi o terceiro mais recorrente no estudo.

Por fim, com apenas 1,80\% cada um, os setores de Tecnologia da Informação e de Telecomunicações foram os menos recorrentes na pesquisa, correspondendo a três empresas cada um. Ressalta-se que o setor Financeiro foi excluído da amostra, pelas razões explicitadas na seção anterior. 
O tom anormal, considerado nesse estudo como proxyde gerenciamento de impressão, foi analisado por setor na Tabela 3. Para tanto, foram calculadas as médias do tom anormal encontrado pela Equação (2). Ressalta-se que, para o cálculo das médias, os valores dos tons anormais foram considerados em termos absolutos.

Tabela 3 - Uso do Tom Anormal por Setor

\begin{tabular}{lc}
\hline Setor & Média TA (NOVO C SETOR) \\
Bens Industriais & 0,1326 \\
Consumo Cíclico & 0,1731 \\
Consumo Não Cíclico & 0,1627 \\
Materiais Básicos & 0,1403 \\
Petróleo, Gás e Biocombustíveis & 0,1669 \\
Saúde & 0,1281 \\
Tecnologia da Informação & 0,1340 \\
Telecomunicações & 0,0617 \\
Utilidade Pública & 0,1417 \\
\hline
\end{tabular}

Fonte: Elaborada a partir dos dados da pesquisa (2018).

A Tabela 3 tem a possibilidade de indicar em quais setores há maior prática, em média, do gerenciamento de impressão por meio do uso do tom anormal. Dessa maneira, nota-se que as empresas classificadas em Consumo Cíclico foram as que mais usaram o tom anormal para gerenciarem a impressão da sua companhia, apresentando uma média absoluta de 0,1731.

Ainda analisando as médias do TA por setor, é possível observar que empresas do setor de Petróleo, Gás e Biocombustiveis tiveram a segunda média mais alta, indicando que essas companhias usam, em média, 0,1669 de tom anormal em seus relatórios de administração. Em contrapartida, segundo a Tabela 3, as empresas dos setores de Telecomunicações e de Saúde foram as que apresentaram as menores médias, sinalizando que as mesmas são as que menos fazem uso do gerenciamento de impressão.

Ainda consoante às análises por setor, a Tabela 4 demonstra a média do uso das palavras classificadas em positivas ou em negativas ao longo dos oito anos observados. 
Tabela 4 - Uso das palavras por setor

\begin{tabular}{lcc}
\hline Setor & Média Palavras Positivas & Média Palavras Negativas \\
Bens Industriais & 79,456 & 36,722 \\
Consumo Cíclico & 76,005 & 32,522 \\
Consumo Não Cíclico & 90,384 & 39,098 \\
Materiais Básicos & 75,271 & 39,844 \\
Petróleo, Gás e Biocombustíveis & 202,583 & 104,000 \\
Saúde & 82,583 & 31,125 \\
Tecnologia da Informação & 68,417 & 25,875 \\
Telecomunicações & 284,625 & 97,625 \\
Utilidade Pública & 118,699 & 72,629 \\
\hline
\end{tabular}

Fonte: Elaborada a partir dos dados da pesquisa (2018).

Segundo a Tabela 4, nota-se que em todos os setores a média das palavras positivas é maior do que a média das negativas. Isso indica que as empresas da amostra, em média, fizeram mais o uso das palavras classificadas em positivas do que as classificadas em negativas. Ressalta-se que somente a análise das médias dessas palavras não é indício de gerenciamento de impressão, visto que a proxy do gerenciamento de impressão desta pesquisa é o tom anormal, obtido pelos resíduos da Equação (2).

Além de terem apresentado a segunda maior média de tom anormal (Tabela 3), as empresas do setor de Petróleo, Gás e Biocombustíveis, em média, foram as que mais fizeram uso das palavras negativas. Cada companhia desse setor usou, em média, 104 palavras negativas ao longo dos oito anos de análise nos seus relatórios de administração. Em relação às palavras positivas, o setor de Telecomunicações apresentou a maior média, de 284.

Também, além de ter apresentado a menor média de gerenciamento de impressão, o setor de Tecnologia da Informação foi o que menos utilizou as palavras classificadas em positivas ou negativas no dicionário da pesquisa. Em média, cada companhia desse segmento usou apenas 68 e 25 palavras positivas e negativas, respectivamente, em seus relatórios ao longo do período analisado.

\subsection{ESTATÍSTICAS DESCRITIVAS DA AMOSTRA}

Conforme abordado na seção dos procedimentos metodológicos, as variáveis explicativas do tom (TOM) são: rentabilidade (RENT), tamanho (TAM), preço da ação/valor patrimonial da ação (P_VPA) e retorno das ações (RET). Ainda, além das variáveis anteriores, também se insere na equação do tom anormal positivo (TAPOS) a variável dummy para perdas (PERDAS). 
Sendo assim, as principais características das variáveis abordadas nesta pesquisa são ilustradas na Tabela 5.

Tabela 5 - Estatística descritiva das variáveis

\begin{tabular}{lccccccc}
\hline & TAM & P_VPA & PERDAS & RENT & RET & TAPOS & TOM \\
Média & 14,320 & 2,149 & - & 1,957 & 0,097 & - & 0,323 \\
Máximo & 20,620 & 71,930 & 1,000 & 449,900 & 3,282 & 1,000 & 1,000 \\
Mínimo & 0,000 & $-25,580$ & 0,000 & $-160,200$ & $-0,958$ & 0,000 & $-1,000$ \\
Desvio-Padrão & 3,407 & 4,202 & 0,450 & 18,270 & 0,507 & 0,500 & 0,290 \\
\hline
\end{tabular}

Fonte: Elaborada a partir dos dados da pesquisa (2018)

Nota: Valores em reais

Percebe-se, de acordo a Tabela 5, que as empresas da amostra apresentaram, em média, valores positivos de potencial de crescimento (P_VPA), de rentabilidade e de retorno das ações. A variável de tamanho TAM, por ser o logaritmo do ativo, somente apresenta valores positivos. As variáveis PERDAS, e TAPOS são variáveis dummies binárias e, por isso, apresentaram valores máximos de 1 e mínimos de 0. O valor baixo de 1,957 é condizente com o indicador utilizado como proxy de rentabilidade ROA (receita líquida/ativo total). 0 valor máximo de 449,9 de rentabilidade foi evidenciado pela empresa AES Elpa, no ano de 2016 e o valor mínimo foi divulgado pela empresa Mendes Jr., em 2017.

Como também foi comentado na seção de procedimentos metodológicos, a variável TOM obtida pela Equação (1), assumiu valores entre -1 e 1 e sua média apresentou valor de 0,323. Além disso, a Tabela 5 indica que a média dos retornos das ações da amostra foi de 0,097. A empresa que obteve o maior retorno de ação $(3,28)$ foi a GPC Part, em 2016. Em contrapartida, no ano de 2015, a companhia PDG Realt apresentou o menor valor de retorno.

Adicionalmente, com o auxílio do programa WordArt, foram criadas duas nuvens de palavras (tag clouds), uma contendo as 15 palavras positivas mais utilizadas pelas empresas da amostra (Figura 1), e a outra com as 15 palavras negativas que mais apareceram nos relatórios (Figura 2).0 objetivo desta técnica consiste em usar tamanhos e fontes de letras diferentes segundo as ocorrências das palavras no texto analisado, sendo que o tamanho da palavra na nuvem é proporcional a sua ocorrência nos textos (DIAS et al., 2014). 


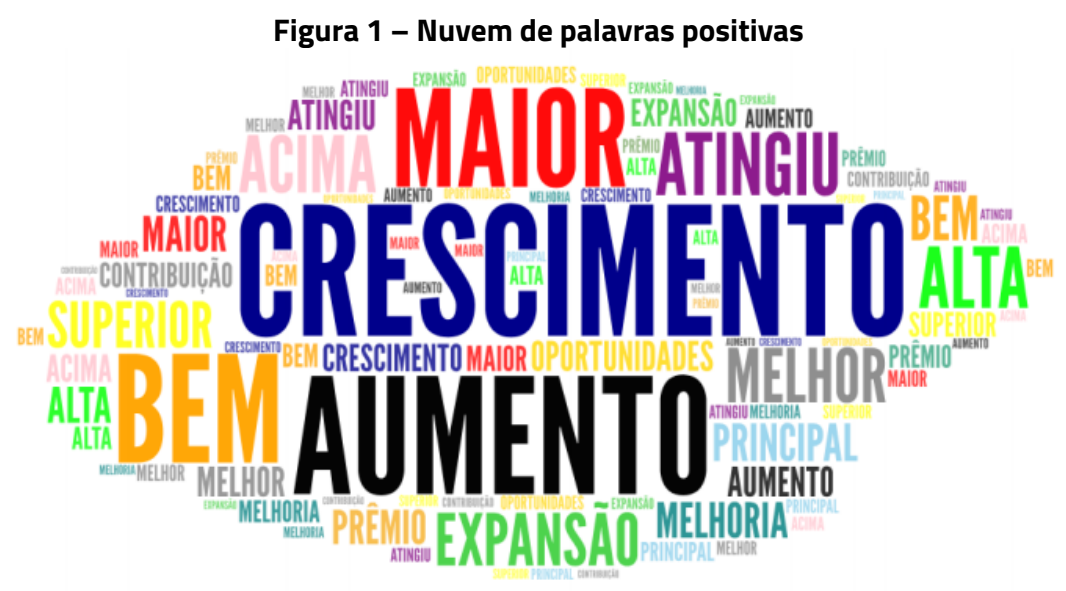

Fonte: Elaborada a partir dos dados da pesquisa (2018)

Dentre as palavras positivas listadas no dicionário da pesquisa, a Figura 1 ilustra as que apareceram com maior recorrência nos relatórios de administração da amostra. Constata-se, portanto, que as companhias centralizaram o foco das suas narrativas, principalmente, na possibilidade de crescimento e, consequentemente, na expansão das mesmas. Também, as palavras "aumento", "maior" e "bem" foram utilizadas com bastante frequência.

Adicionalmente, termos que se referem ao cumprimento de metas e aperfeiçoamento das empresas também tiveram destaque, como "atingiu", "oportunidades" e "contribuição". Nota-se que a maioria das palavras realçadas demonstra características e ações esperadas das empresas, corroborando a teoria de Hines (1988), de que as companhias procuram passar a imagem esperada pela sociedade, em busca de credibilidade e legitimação.

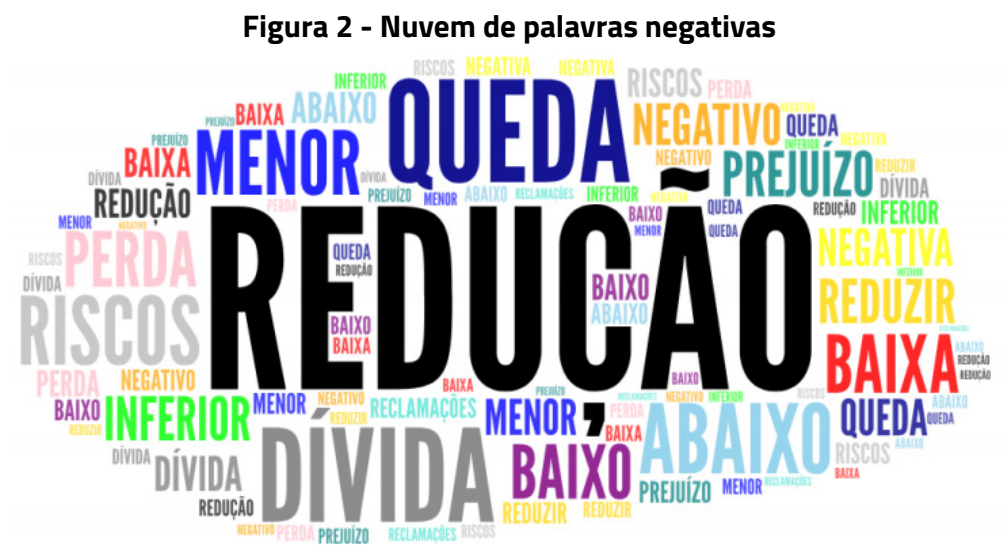

Fonte: Elaborada a partir dos dados da pesquisa (2018) 
Por outro lado, a Figura 2 demonstra as 15 palavras mais utilizadas, classificadas. É possivel inferir que a nuvem de palavras da Figura 5 apresenta os termos que representam, em regra, os receios dos administradores de empresas em geral, como "queda", "dívida", "baixa", "riscos", "perda" e "prejuízo". É interessante ressaltar também a grande utilização da palavra "reclamações", que indica a insatisfação, seja por parte dos membros da companhia ou de outros stakeholders.

\subsection{DETERMINANTES DO GERENCIAMENTO DE IMPRESSÃO}

\subsubsection{Determinantes do tom total}

Conformejá explicitado nos procedimentos metodológicos, a $\mathrm{H}_{1}$ que busca analisar o comportamento do tom total em relação ao desempenho financeiro, foi testada por meio da Equação (2). Para tanto, os testes F de Chow (1960), de Hausman (1978) e LM de Breusch e Pagan (1980) foram realizados para a escolha do método mais adequado aos modelos da pesquisa (Tabela 6).

\begin{tabular}{lll}
\multicolumn{2}{c}{ Tabela 6 - Resultados dos testes de validação dos modelos para estimação } \\
\hline \multicolumn{1}{c}{ Testes } & Valor das Estatísticas dos testes & Modelo Escolhido \\
Teste de Chow & Prob $>\mathrm{F}=0.0000$ & Efeitos Fixos \\
Teste de Breusch-Pagan (LM) & Prob $>\chi^{2}=0.000$ & Efeitos Aleatórios \\
Teste de Hausman & Prob $>\chi^{2}=0.8795$ & Efeitos Aleatórios \\
\hline
\end{tabular}

Fonte: Elaborada a partir dos dados da pesquisa (2018)

Após a realização dos testes, concluiu-se que o método mais apropriado para a aplicação da Equação (2) é o modelo de dados em painel com efeitos aleatórios. A Tabela 7 evidencia os resultados encontrados a partir da regressão rodada. 
Tabela 7 - Resultado das Equações (2)

\begin{tabular}{lcccc}
\hline Variável & Coeficiente & Desvio-Padrão & Estatística-T & P-Valor \\
\hline C & 0,502 & 0,045 & 11,172 & $0,000^{* * *}$ \\
Rent & 0,007 & 0,001 & 9,244 & $0,000^{* * *}$ \\
Ret & 0,021 & 0,014 & 1,510 & 0,132 \\
Tam & $-0,013$ & 0,002 & $-5,662$ & $0,000^{* * *}$ \\
P_vpa & 0,014 & 0,003 & 5,230 & $0,000^{* * *}$ \\
Util pública & $-0,124$ & 0,044 & $-2,805$ & $0,005^{* * *}$ \\
Telecomun & 0,134 & 0,084 & 1,599 & 0,110 \\
Tecno da info & 0,083 & 0,100 & 0,836 & 0,404 \\
Saúde & 0,050 & 0,058 & 0,858 & 0,391 \\
Petro, gas e bio & 0,071 & 0,086 & 0,822 & 0,412 \\
Materiais básicos & $-0,076$ & 0,046 & $-1,635$ & 0,102 \\
Consumo não cíclico & $-0,016$ & 0,055 & $-0,284$ & 0,776 \\
Consumo cíclico & $-0,006$ & $\mathbf{R}^{2}$ & 0,038 & $-0,152$ \\
\hline Observações & 1336 & 0,227 & 0,879 \\
Durbin Watson & 1,471 & & & \\
\hline
\end{tabular}

Fonte: Elaborada a partir dos dados da pesquisa (2018)

\section{Nota: Niveis de significância: ${ }^{*} p<0,10 ;{ }^{* *} p<0,05 e^{* * *} p<0,01$}

Segundo a Tabela 7, observa-se que o poder de explicação do modelo é de 0,227, indicando que o modelo explica mais de $20 \%$ do tom total. Ainda, ao comparar o valor de $\mathrm{R}^{2}$ encontrado na pesquisa e o valor apresentado por Carlsson e Lamti (2015) - $R^{2}$ de 0,2921, observa-se a semelhança nos seus valores.

Ao analisar a relação entre as variáveis, conclui-se que alguns resultados se mostraram condizentes aos da literatura. Assim como no estudo de Carlsson e Lamti (2015), as variáveis RENT e TAM apresentaramse com significância estatística, ao nível de 1\%. Também, o valor do coeficiente de RENT apresentou o mesmo sinal que foi apresentado pelas autoras. Contudo, o coeficiente de TAM apresentou sinal negativo, diferentemente das autoras. Dessa maneira, a Tabela 7indica que empresas com melhor desempenho financeiro fazem mais uso de tom nas suas narrativas contábeis, enquanto empresas maiores fazem menos uso do tom.

Ainda, assim como ocorreu na pesquisa de Carlsson e Lamti (2015), a variável representativa do potencial de crescimento P_VPA demonstrou significância estatística no estudo. Também, o sinal do coeficiente dessa variável foi diferente do sinal apresentado por Carlsson e Lamti (2015). Porém, essa 
diferença é coerente e indica resultados similares, tendo em vista que as pesquisadoras utilizaram a variável Book to Market - BTM, que é o inverso da utilizada na presente pesquisa. O único setor que se mostrou explicativo no modelo foi o de Utilidade Pública, evidenciando que companhias desse setor usam menos o tom nos seus relatórios. Além disso, a estatística Durbin Watson na Equação (2) indica a existência de uma autocorrelação positiva entre os resíduos, pois o seu valor foi de 1,471.

Ante o exposto, $\mathrm{a}_{1}$ (o tom nos relatórios de administração é positivamente associado à performance financeira da empresa) não pode ser rejeitada pela Equação (2). Isso se dá, pois, além da variável RENT ter demonstrado significância estatística, o seu coeficiente apresentou valor positivo, bem como era esperado pela $\mathrm{H}_{1}$. $\mathrm{O}$ resultado da Equação (2) foi similar ao estudo de Carlsson e Lamti (2015), que também não puderam rejeitar a hipótese de relação entre as variáveis.

\subsubsection{Determinantes do tom anormal positivo}

Para testar a $\mathrm{H}_{2^{\prime}}$ a Equação (3) foi executada a fim de aceitá-la ou rejeitá-la. Ressalta-se que, como o valor do tom anormal foi encontrado por meio dos resíduos da regressão2, o tom anormal positivo (variável dependente dessa equação) representa uma variável dummy do tom anormal.

Como a variável dependente do tom anormal positivo (TAPOS) das equações é uma variável dummy, isto é, assume o valor de 1 ou 0, a regressão logística é a mais adequada para a aplicação dos modelos (CARLSSON; LAMTI, 2015). Dessa forma, a Equação (3) estima a probabilidade associada à ocorrência do uso do tom anormal positivo em face do conjunto das variáveis explanatórias das equações. Assim sendo, a Tabela 8 detalha o resultado da equação. 
Tabela 8 - Resultado das Equações (3)

\begin{tabular}{lcccc}
\hline Variável & Coeficiente & Desvio-Padrão & Estatística-T & P-Valor \\
C & 0,752 & 0,376 & 1,998 & 0,046 \\
Rent & $-0,024$ & 0,019 & $-1,296$ & 0,195 \\
Rent_perdas & 0,014 & 0,021 & 0,677 & 0,498 \\
Perdas & $-0,604$ & 0,223 & $-2,705$ & $0,007^{* * *}$ \\
Ret & $-0,103$ & 0,147 & $-0,700$ & 0,484 \\
P_vpa & $-0,021$ & 0,023 & $-0,921$ & 0,357 \\
Logtam & $-0,016$ & 0,022 & $-0,705$ & 0,481 \\
Util pública & $-0,170$ & 0,268 & $-0,634$ & 0,526 \\
Telecomun & $-0,644$ & 0,478 & $-1,347$ & 0,178 \\
Tecno da info & $-0,114$ & 0,554 & $-0,206$ & 0,836 \\
Saúde & $-0,204$ & 0,341 & $-0,600$ & 0,549 \\
Petro,gas e bio & $-0,428$ & 0,520 & $-0,822$ & 0,411 \\
Materiais básicos & $-0,150$ & 0,275 & $-0,547$ & 0,584 \\
Consumo cíclico & 0,022 & 0,230 & 0,098 & 0,922 \\
Consumo não cíclico & $-0,135$ & 0,310 & $-0,434$ & 0,664 \\
\hline Observações & 1336 & & & \\
McFadden R & 0,013 & & & \\
\hline
\end{tabular}

Fonte: Elaborada a partir dos dados da pesquisa (2018) Nota: Níveis de significância: ${ }^{*} p<0,10 ;{ }^{* *} p<0,05 e^{* * *} p<0,01$

Observa-se na Tabela 8 que somente a variável dummy PERDAS pode ser considerada como variável explicativa do uso do tom anormal positivo. Enfatiza-se que PERDAS assumiu o valor 1 quando a rentabilidade apresentou valor negativo e valor 0 quando a rentabilidade foi positiva. Em relação ao valor de McFadden $R^{2}$, nota-se que ele apresentou valor baixo na Equação (3) (de 0,013). Porém, esse valor ainda foi maior do que o encontrado por Carlsson e Lamti (2015), que mostrou um $\mathrm{R}^{2}$ de 0,010. Porém, assim como ocorreu na Equação (2), os valores de $\mathrm{R}^{2}$ dos estudos apresentaram valores próximos.

É possivel observar que o coeficiente da variável PERDAS apresentou sinal negativo. Como a regressão rodada foi logística, isso indica que foi evidenciado que a probabilidade de se observar o uso de um tom anormal positivo nos relatórios de administração diminui conforme o desempenho financeiro das empresas diminui. Apesar da variável PERDAS ter demonstrado significância estatística nos resultados do modelo, conclui-se que a $\mathrm{H}_{2}$ (a probabilidade de se observar o uso de um tom anormal positivo nos 
relatórios de administração aumenta conforme o desempenho financeiro das empresas diminui) pode ser rejeitada, pois para não rejeitá-la, o sinal do coeficiente deveria ter sido positivo. Carlsson e Lamti (2015) também rejeitaram a hipótese, porém, em sua pesquisa, a rejeição ocorreu, pois, PERDAS não se mostrou com significância estatística, por isso, as autoras não puderam inferir sobre seu comportamento perante o tom anormal positivo.

\subsubsection{Teste de correlação de Pearson e Variance Inflation Factor}

O objetivo da análise de correlação de Pearson é explorar a correlação entre duas variáveis e detectar possíveis problemas de multicolinearidade. Dessa maneira, as variáveis utilizadas nas Equações (2) e (3) foram correlacionadas e a Tabela 9 evidencia o resultado da relação entre elas.

Tabela 9 - Correlação de Pearson

\begin{tabular}{lccrrrrr}
\hline & TAPOS & RENT & PERDAS & TAM & P_VPA & RET & TOM \\
\hline TAPOS & 1 & & & & & & \\
RENT & 0,007 & 1 & & & & & \\
PERDAS & $-0,147$ & $-0,404$ & 1 & & & & \\
TAM & $-0,022$ & 0,069 & 0,013 & 1 & & & \\
P_VPA & 0,024 & 0,141 & $-0,171$ & 0,030 & 1 & \\
RET & 0,004 & 0,162 & $-0,230$ & $-0,071$ & 0,105 & 1 & \\
TOM & 0,691 & 0,126 & $-0,334$ & $-0,146$ & 0,210 & 0,163 & \\
\hline
\end{tabular}

Fonte: Elaborada a partir dos dados da pesquisa (2018)

A Tabela 9 mostra que o modelo da regressão não possui problema de multicolinearidade, pois os valores dos coeficientes de Pearson apresentaram valores baixos. Observa-se, no entanto, que a variável TOM apresentou coeficientes de relação altos com e TAPOS, porém, esse valor é esperado, visto que TAPOS são os resíduos positivos de TOM. Porém, isso não é um problema, pois essas variáveis não são usadas simultaneamente nas equações.

Também, o valor de -0,404 do coeficiente entre RENT e PERDAS já era esperado, pois PERDAS é uma variável dummy baseada em RENT. Como as duas variáveis são usadas simultaneamente na Equação (3), poderia haver um problema de colinearidade. Por esse motivo, a variável de interação RENT_PERDAS usada na Equação (3) se justifica para sanar esse possivel problema (CARLSSON; LAMTI, 2015).

Outro método para detectar possíveis problemas de multicolinearidade é o Variance Inflation Factor - VIF, que, neste estudo, foi realizado para dar mais robustez aos resultados. A Tabela 10 detalha os valores de VIF encontrados. 


\section{Tabela 10 - Teste Variance Inflation Factor}

\begin{tabular}{cc}
\hline Variáveis & VIF \\
\hline RENT & 1,2798 \\
PERDAS & 1,3091 \\
TAM & 1,0148 \\
P_VPA & 1,0429 \\
RET & 1,0772 \\
\hline
\end{tabular}

Fonte: Elaborada a partir dos dados da pesquisa (2018)

Segundo May et al. (2018), valores de VIF acima de 5 podem indicar uma alta relação linear e problemas de multicolinearidade. Tendo como base esse parâmetro, segundo os valores apresentados na Tabela 10, os modelos das Equações (2) e (3) não apresentaram problema de multicolinearidade, conclusão obtida também pelo método de correlação de Pearson (Tabela 9).

\section{CONSIDERAÇÕES FINAIS}

A presente pesquisa teve como objetivo analisar as características do gerenciamento de impressão por meio do uso de tom anormal nos relatórios de administração das companhias abertas listadas na Brasil, Bolsa, Balcão [B] ${ }^{3}$, durante os anos de 2010 a 2017. Nesse sentido, 1.576 relatórios foram baixados manualmente e analisados com o auxílio do software MAXQDA 12. Para o atingimento do objetivo do estudo, realizou-se uma pesquisa com abordagem do problema quantitativa, por meio documental, com análise descritiva dos dados.

Verificou-se que a amostra da pesquisa foi composta, principalmente, por empresas do setor de Consumo Cíclico. Ainda, os setores que apresentaram maior média de TA e, consequentemente, o maior nível de gerenciamento de impressão, foram o de Consumo Cíclico e o de Petróleo, Gás e Biocombustíveis. Ainda, observou-se que, em média, as companhias usam mais palavras positivas do que negativas em suas narrativas contábeis.

Notou-se, também, que as empresas da amostra apresentaram no período analisado, em média, desempenho financeiro e potencial de crescimento positivos. Em relação às palavras do dicionário, as negativas mais utilizadas foram "queda", "dívida", "baixa", "riscos", "perda" e "prejuízo", que retratam receios comuns de todos os empresários.

Ainda, por meio da nuvem de palavras positivas, foi evidenciado que as companhias centralizaram o foco das suas narrativas, principalmente, na sua possibilidade de crescimento. Adicionalmente, foi 
observado que as palavras positivas demonstram características e ações esperadas das empresas, corroborando a teoria de Hines (1988), de que as companhias procuram passar a imagem esperada pela sociedade, em busca de credibilidade e legitimação.

Em relação aos modelos rodados, encontrou-se que empresas com melhor desempenho financeiro e maior potencial de crescimento fazem mais uso de tom nas suas narrativas contábeis. Dessa forma, a Hipótese $\mathrm{H}_{1}$ de que o tom nos relatórios de administração estaria positivamente associado à performance financeira da empresa não pode ser rejeitada pela pesquisa. Carlsson e Lamti (2015), estudo no qual a presente pesquisa se baseou, também não puderam rejeitar a hipótese.

Em relação ao tom anormal positivo, proxy para o gerenciamento de impressão positivo, foi evidenciado que a única variável explicativa do tom anormal positivo é a representativa de perdas. Contudo, os resultados do estudo indicaram que a probabilidade de se observar o uso de um tom anormal positivo nos relatórios de administração diminui conforme o desempenho financeiro das empresas diminui. Por isso, a Hipótese $\mathrm{H}_{2}$ de que a probabilidade de se observar o uso de um tom anormal positivo nos relatórios de administração aumentaria conforme o desempenho financeiro das empresas diminui foi rejeitada pelas duas equações, assim como na pesquisa de Carlsson e Lamti (2015).

Espera-se, dessa forma, que o presente trabalho permita um maior conhecimento sobre essa prática de gerenciamento e, dessa forma, os usuários consigam detectá-la mais facilmente. Com isso, também se espera contribuir para que as empresas tenham menos incentivos para fazer uso dessa prática, tendo em vista que, apesar de a mesma não ser ilegal, pode levar os usuários a tomar decisões equivocadas. Ainda, o dicionário de palavras positivas e negativas da pesquisa também é uma contribuição da mesma, tendo em vista que ela já passou pelo processo de validação de profissionais e pode ser utilizada em pesquisas nacionais futuras.

Tendo em vista que a pesquisa se limitou a analisar o gerenciamento de resultados somente nas narrativas contábeis, sugere-se, para pesquisas futuras, que a prática também seja testada em relação a distorções em gráficos. Também, como o estudo contou com uma amostra grande, não foi possível analisar cada relatório manualmente. Dessa forma, também é sugerido, para futuros estudos, que a quantidade da amostra diminua e, dessa maneira, seja realizada uma análise minuciosa e manual de cada relatório de administração.

\section{REFERÊNCIAS}

BEATTIE, V.; JONES, M. J. Impression management: the case of inter-country financial graphs. Journal of International Accounting, Auditing \& Taxation, [S.I.], v. 9, n. 2, p. 159-183, 2000. 
BEATTIE, V.; JONES, M. J. The use and abuse of graphs in annual reports: a theoretical framework and empirical study. Accounting and Business Research, [S.I.], v. 22, n. 88, p. 291-303, 1992.

BONFIM, M. P.; SOUZA, I. G. M.; SILVA, C. A. T.; SERAFIM, A. O. Gerenciamento de impressão gráfica no processo decisório. Revista Ambiente Contábil, Natal, v. 10, n. 1, p. 71-87, 2018.

BOZZOLAN, S.; CHO, C. H.; MICHELON, G. Impression Management and Organizational Audiences: The Fiat Group Case. Journal of Business Ethics, [S.I.], v. 126, n. 1, p. 143-165, 2015.

BRENNAN, N.; SAORIN, E. G.; PIERCE, A. Impression management: developing and illustrating a scheme of analysis for narrative disclosures - a methodological note. Accounting, Auditing \& Accountability Journal, [S.I.], v. 22 n. 5, p.789-832, 2009.

BREUSCH, T. S.; PAGAN, A. R. The lagrange multiplier test and its applications to model specification in econometrics. Review of Economic Studies, [S.I.], v. 47, n. 1, p. 239-253, 1980.

CARLSSON, S.; LAMTI, R. Tone management and earnings management: a UK evidence of abnormal tone in CEO letters and abnormal accruals. 2015. 54 f. Thesis (Master in Accounting) - School of Business, Economics and Law, University of Gothenburg, Gothenburg, 2015.

CEN, Z.; CAl, R. Impression management in Chinese corporations: a study of chairperson's statements from the most and least profitable Chinese companies. Asia Pacific Business Review, [S.I.], v. 19, n. 4, p. 490-505, 2013.

CHO, C. H.; MICHELON, G.; PATTEN, D. Impression Management in Sustainability Reports: An Empirical Investigation of the Use of Graphs. Accounting and the Public Interest, [S.I.], v. 12, n. 1, p. 16-37, 2012.

CHOW, G. C. Tests of equality between sets of coefficients in two linear regressions. Econometrica, [S.I.], v. 28, n. 3, p. 591-605, 1960.

CLATWORTHY, M. A.; JONES, M. J. Financial Reporting of Good News and Bad News: Evidence from Accounting Narratives. Accounting and Business Research, [S.I.], v. 33, n.3, p. 171-185, 2003.

COLAUTO, R. D.; BEUREN, I. M. Coleta, análise e interpretação dos dados. In: BEUREN, I. M. (Org.). Como elaborar trabalhos monográficos em contabilidade: teoria e prática. 3. ed. São Paulo: Atlas, 2014. p. 117-144.

CUNHA, R. K. C.; SILVA, C. A. T. Análise da Facilidade de Leitura das Demonstrações Contábeis das Empresas Brasileiras: uma investigação do gerenciamento de impressões nas narrativas contábeis. In: Congresso USP de Controladoria e Contabilidade, 9, 2009. Anais... São Paulo: FEA/USP, 2009. 
DAVIS, A. K.; TAMA-SWEET, I. Managers' use of language across alternative disclosure outlets: earnings press releases versus MD\&A. Contemporary Accouting Research, [S.I.], v. 29, n. 3, p. 804-837, 2012.

DIAS, M. S.; PARENTE, J. R.; VASCONCELOS, M. I.; DIAS, F. A. Intersetorialidade e Estratégia Saúde da Família: tudo ou quase nada a ver? Ciência \& Saúde Coletiva, [S.I.], v. 19, n. 11, p. 4371-4382, 2014.

DOMINGOS, S. R. M.; PONTE, V. M. R.; PAULO, E.; ALENCAR, R. C. Gerenciamento de resultados contábeis em oferta pública de ações. Revista Contemporânea de Contabilidade, Florianópolis, v. 14, n. 31, p. 89107, jan./abr. 2017.

FALSCHLUNGER, L.; EISL, C.; LOSBICHLER, H.; GREIL; A. M. Impression management in annual reports of the largest European companies, Journal of Applied Accounting Research, [S.I.], v. 16, n. 3, p. 383-399, 2015.

GIL, Antonio Carlos. Métodos e técnicas de pesquisa social. 6. ed. São Paulo: Atlas. 2008.

HAUSMAN, J.A. Specification tests in econometrics. Econometrica, [S.I.], v. 46, n. 6, p. 1251-1271, 1978.

HENRY, E. Are investors influenced by how earnings press releases are written? Journal of Business Communication, [S.I.], v. 45, n. 4, p. 363-407, 2008.

HINES, R. D. Financial accounting: in communicating reality, we construct reality. Accounting. Organizations and Society, [S.I.], v. 13, n. 3, p. 251-261, 1988.

HUANG, X.; TEOH, S.; ZHANG, Y. Tone management. The Accouting Review, [S.I.], v. 89, n. 3, p. 10831113, 2014.

JONES, M. J. The nature, use and impression management of graphs in social and environmental Accounting. Accounting forum, [S.I.], v. 35, n. 2, p. 75-89, 2011.

LI, F. The information content of forward-looking statements in corporate filings - a naïve bayesian machine learning approach. Journal of Accounting Research, [S.I.], v. 48, n. 5, p. 1049-1102, 2010.

MAIOR, C. D. S.; FERREIRA, A. F.; SOUZA, A. C.; BORBA, J. A. Gerenciamento de Impressão de Gráficos: Uma Análise Experimental de Investimentos com Estudantes de Graduação. In: Encontro da ANPAD, XXXVI, Rio de Janeiro, 2012. Anais... Rio de Janeiro: ANPAD, p. 1-11, 2012.

MARTINS, G. A. Manual para elaboração de monografias e dissertações. 3. ed. São Paulo: Atlas, 2013. 
MATHER, D.; MATHER, P.; RAMSAY, A. An investigation into the measurement of graph distortion in financial reports. Accounting and Business Research, [S.I.], v. 35, n. 2, p. 147-160, 2005.

MAY, J. R.; VISENTAINER, J.; CUNHA, L. C. Valor percebido e perfil dos alunos do curso de Ciências Contábeis. Revista Brasileira de Contabilidade, [S.I.], n. 232, p. 70-81, ago. 2018.

MENDONÇA, J. R.C.; AMANTINO-DE-ANDRADE, J. Gerenciamento de impressões: em busca da legitimidade organizacional. Revista de Administração de Empresas, [S.I.], v. 43, n.1, p. 36-48, 2003.

MERKL-DAVIES, D. M.; BRENNAN, N. Discretionary disclosure strategies in corporate narratives: incremental information or impression management? Journal of Accounting Literature, [S.I.], v. 26, p. 116196, 2007.

MICHELON, G. Impression management and legitimacy strategies: The BP case. Financial Reporting, [S.I.], v. 4, p. 35-64, 2012.

MIRANDA, L.C.; VIEIRA, A. S.; LAGIOIA, U.C.T.; VASCONCELOS, M.T.C. Utilização de gráficos em demonstrações contábeis. Revista de Educação e Pesquisa em Contabilidade, [S.I.], v. 2, n. 3, p. 16-35, 2008.

PATELLI, L.; PEDRINI, M. Is the Optimism in CEO's Letters to Shareholders Sincere? Impression Management Versus Communicative Action During the Economic Crisis. Journal of Business Ethics, [S.I.], v. 124, n. 1, p. 1-16, 2014.

PENTEADO, I. A. M. Gerenciamento de impressão em relatórios de sustentabilidade no Brasil: uma análise do uso de gráficos. 116f. Dissertação (Mestrado em Controladoria e Contabilidade) - Faculdade de Economia, Administração e Contabilidade de Ribeirão Preto, Universidade de São Paulo, Ribeirão Preto, 2013.

RAUPP, F. M.; BEUREN, Ilse Maria. Metodologia da pesquisa aplicável às ciências sociais. In: BEUREN, I. M. (Org.). Como elaborar trabalhos monográficos em contabilidade: teoria e prática. 3. ed. São Paulo: Atlas, 2014. p. 76-97.

RUTHERFORD, B. A. Genre analysis of corporate annual report narratives: a corpus-linguistic based approach. Journal of Business Communication, [S.I.], v. 42, n. 4, p. 349-378, 2005.

SAORIN, E. G.; ISIDRO, H.; MARQUES, A. Impression Management and Non GAAP Disclosure in Earnings Announcements. Journal of Business Finance \& Accounting, [S.I.], v. 44, n. 3-4, p. 448-479, 2017.

SAORIN, E. G.; OSMA, B. G. Self-serving financial reporting communication: A study of the association between earnings management and impression management. [S.I.], Aug. 2010. Disponivel em: http://ci- 


\section{Gestãoe \\ Desenvolvimento}

e-ISSN: 2446-6875

p-ISSN: $1807-5436$

teseerx.ist.psu.edu/viewdoc/download?doi=10.1.1.463.3003\&rep=rep1\&type=pdf. Acesso em: 17 ago. 2020.

SCHLENKER, B. R. Impression management: the self-concept, social identity, and interpersonal relations. Monterrey: Brooks/Cole, 1980.

SEVERINO, A. J. Metodologia do trabalho científico. 23. ed. São Paulo: Cortez, 2007.

SILVA, C. A. T.; RODRIGUES, F. F.; ABREU, R. L. Análise dos Relatórios de Administração das Análise dos Relatórios de Administração das Companhias Abertas Brasileiras: um Estudo do Exercício Social de 2002. Revista de Administração Contemporânea, [S.I.], v. 11, n. 2, p. 71-92, 2007.

SOUZA, A. C. As palavras importam? 0 uso do tom linguístico nos discursos das apresentações de resultados. 163f. Tese (Doutorado em Ciências) - Faculdade de Economia, Administração e Contabilidade, Universidade de São Paulo, São Pulo, 2017.

WOOLDRIDGE, J. Econometric Analysis of Cross Section and Panel Data. Massachusetts: MIT Press, 2002. 